\title{
Recognizing Interaction from a Robot's Perspective
}

\author{
Tamie Salter*†, François Michaud*, Kerstin Dautenhahn ${ }^{\dagger}$, Dominic Létourneau* and Serge Caron* \\ ${ }^{*}$ LABORIUS - Research Laboratory on Mobile Robotics and Intelligent Systems \\ Department of Electrical Engineering and Computer Engineering \\ Université de Sherbrooke, Sherbrooke, Québec, Canada, J1K 2R1 \\ Email:\{tamie.salter, francois.michaud, dominic.letourneau, serge.caron\}@USherbrooke.ca \\ ${ }^{\dagger}$ Adaptive Systems Research Group, School of Computer Science \\ University of Hertfordshire, Hatfield, United Kingdom, AL10 9AB \\ Email: k.dautenhahn@herts.ac.uk
}

\begin{abstract}
For meaningful interaction between a robot and a human, an autonomous robot must recognize whether the experienced situation is created by people or by the environment. Using only proprioceptive data from a mobile robotic platform, we discover that it is possible to distinguish sensory data patterns involving interaction. These patterns are obtained whilst navigating varying environments, both human populated and unpopulated. The paper reports the initial set of trials using Roball, a spherical mobile robot. Also described is the experimental methodology currently followed to validate the hypothesis that child interaction can be perceived directly from navigation sensors onboard a robotic platform.
\end{abstract}

Index Terms - Human-Robot Interaction (HRI), Adaptive Mobile Robots, Recognizing Interaction, Sensor Evaluation.

\section{INTRODUCTION}

An important capability for an interactive robot is to be able to recognize when it is receiving interaction from people, compared to when it is simply navigating in the world. Humanrobot interaction (HRI) is a growing field with researchers increasingly looking at how children interact with and perceive robots [1], [2], [3], [4], [5]. Achieving natural communication or interaction within the field, of HRI (children or otherwise) is still a long way off. Current popular forms of interaction involve sophisticated sensing, such as vision and audio processing (e.g. [4], [5], [6], [7]), but at a high cost in sensing equipment, energy consumption and processing power. Some systems utilize buttons that must be pushed in order to register touch or communication from people (e.g. [6], [8], [9]). People working with children or in therapy are beginning to recognize that natural touch is an important form of interaction or communication with a robot (e.g. [1], [7], [10], [11]). In previous work [10], [11], [12], it was shown that infrared sensors, usually exploited for navigation purposes, can be used to record interactions or natural touch coming from children playing with a mobile robot. The research demonstrates that it is even possible to detect personality traits (e.g., boisterous, or cautious) of a child interacting with a wheeled robot, simply from the analysis of infrared sensor data.

To demonstrate that such capability can be seen on a different robotic platform with other types of proprioceptive or navigation sensors, we have tested the principle on a spherical robotic ball named Roball [13]. The objective is to see if, through the analysis of Roball's sensor readings, it is

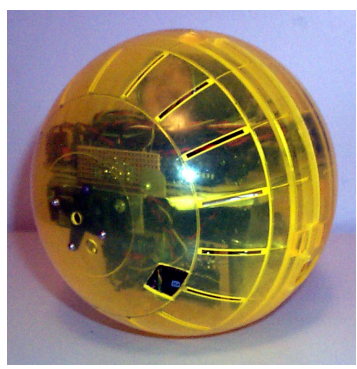

Fig. 1. Roball, the autonomous mobile robot used in the trials.

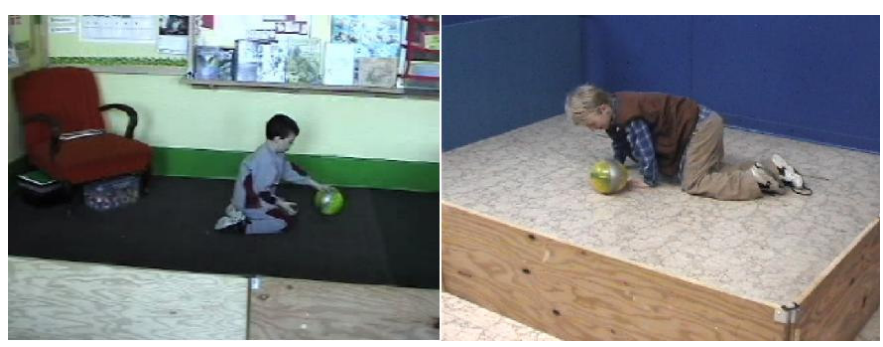

Fig. 2. Pictures of children playing with Roball. In a school environment (left) and at a play group (right).

possible to recognize the environmental conditions the robot is experiencing, whilst in a human populated setting. More specifically, the research question we are studying is: can we distinguish from sensor readings whether the robot is receiving interaction from humans, and what type of interaction is the robot receiving (e.g., being carried, being pushed, receiving no interaction, etc.)? To answer this question, we systematically tested and analyzed Roball's sensory readings taken during controlled laboratory experiments. The preliminary knowledge gained from these experiments is utilized and applied in the analysis of the children-robot trials which have been conducted in a school environment and also in a play group setting. In this paper we describe the systematic investigation of the readings produced by the accelerometers and tilt sensors onboard Roball and the results obtained. Also briefly discussed are the positive preliminary results from the child-robot study we have conducted, and a planned trial where we hope to use results to adapt the robots behavior to the child that is 
interacting with it.

\section{EXPERIMENTAL SETUP}

Shown in Fig. 1, Roball is 6 inches in diameter and weighs about 4 pounds. It consists of a plastic sphere (a hamster exercise ball) constructed from two halves that are attached to each other. The plastic sphere is used to house the fragile electronics (sensors, actuators, processing elements), thus making it robust and ideal for interaction with children. The robot is equipped with three accelerometers, one for each axis (X, Y and $\mathrm{Z}$ ), and three tilt sensors, one for left tilt, one for right and one for forward/backward tilt. Analog ADXL311 miniature accelerometer devices are used to measure Roball's acceleration up to $\pm 2 g$ providing the three axis readings. Sharp GP1S036HEZ miniature photointerrupters are used to detect tilt direction caused by gravity. Tilt sensors are positioned on Roball's printed circuit board (PCB) to detect the internal plateau's front-back and left-right position inside the robot's shell. Two tilt sensors are placed on the left-right axis (the axis corresponding to the line between Roball's two propulsion motors). These are positioned in a symmetrical manner on each side from the center of the left-right axis. This configuration allows the detection of either left or right tilt with both sensors giving the same value, and also allows detection of rotation with readings from the sensors giving opposite left-right tilt values due to centrifuge acceleration.

The motion produced by this platform creates interesting interplay situations. Roball's programming generates what can be termed "catch-and-grab" cycles: during these trials the robot wanders randomly around in the environment, moving away from obstacles sensed from the horizontal angle of the internal plateau. Once the robot detects an object, it reverses for approximately half a meter and then it changes its direction to the right by moving its counterweight to the -30 degrees position ( 0 degree being perpendicular to the plateau). It then proceeds in a random forward motion again. The speed is set to approximately a $\frac{1}{4}$ meter per second for both the forward and backward motion (moderately slow, this is half the maximum speed). This behavior is carried out for the duration of the trial. At the end of the trial, the robot stops by itself. The experimental arena is sectioned off by small wooden walls which creates a pen, as shown in Fig. 3. The pen is approximately $2.5 \mathrm{~m} \times 2 \mathrm{~m}$. The trials were broken down into the seven experimental conditions listed below.

1) Alone $\mathbf{i}$ - Wandering in a laboratory's normal environment with desks and chairs present (no pen or humans present).

2) Alone ii - Roball wandering in the pen by itself, no objects or humans present.

3) Light boxes - Light boxes were placed in the pen to create obstacles that can be pushed by the robot and move (no humans present).

4) Heavy boxes - Heavy boxes were placed in the pen to create fixed obstacles that cannot be pushed by the robot (no humans present).

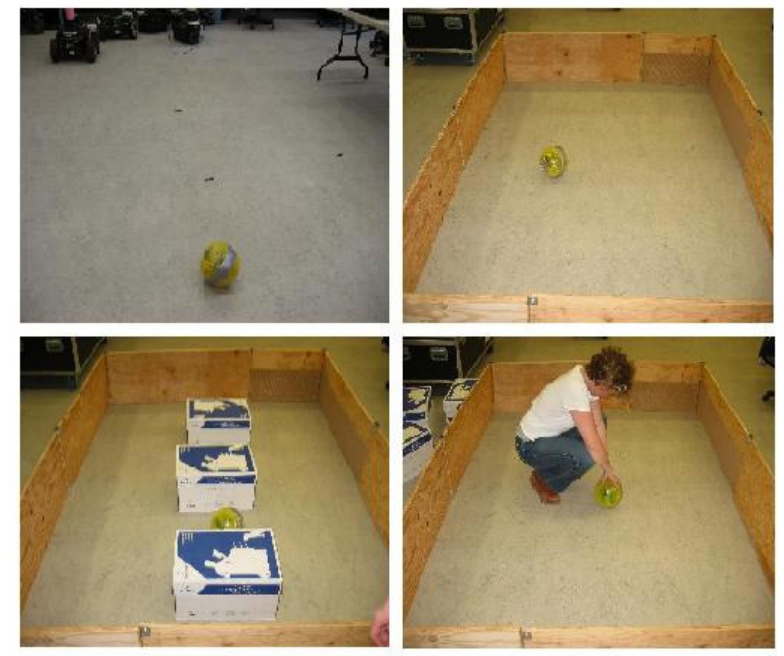

Fig. 3. Pictures giving examples of the different environmental conditions used for the trials. The robot carried out simple obstacle avoidance. The top left is the setting for (1) Alone i, top right is the setting for (2) Alone ii, bottom left is the setting for (3) Light boxes and (4) Heavy boxes, and bottom right is the setting (7) Spinning with the experimenter spinning the robot.

5) Carrying - Experimenter carrying Roball whilst walking for the duration of the experiment.

6) Interaction - Experimenter using her feet and hands to simulate interaction from a child pushing, banging and getting in the way of the robot whilst in the pen.

7) Spinning - Experimenter purposely spinning the robot for the duration of the experiment within the pen.

Three separate experiments were conducted for each of the seven conditions and each individual experiment lasted for a duration of five minutes (this is the approximate time our child-robot trials are conducted for). Thus, in total, 21 experiments were carried out, lasting a total of 105 minutes.

\section{DATA ANALYSIS}

The data on interactions is obtained from the Roball's sensor readings. These sensor readings are memorized onboard the robot 10 times per second. After each experiment, the data is downloaded from the robot to a computer. The records of the two different sets of sensor data are then analyzed to investigate which data can be recognized as interaction patterns.

Tilt sensors - Investigated were the different values produced by these sensors. Of interest was whether, as expected, it was possible to tell when Roball was spinning from the readings produced by the tilt sensors. This should be evident when the two different tilt sensors produce different readings. This should occur when the $\mathrm{G}$ force created by the robot being spun pushes the tilt sensors to opposite sides of left/right axis, thus given opposite readings. The difference between the right and left tilt sensors for each data log, along with the overall sum of this difference are analyzed. In previous work with Roball, Michaud et al. [13] utilized mercury tilt switches to detect spinning, whilst the robot was still (not moving). 


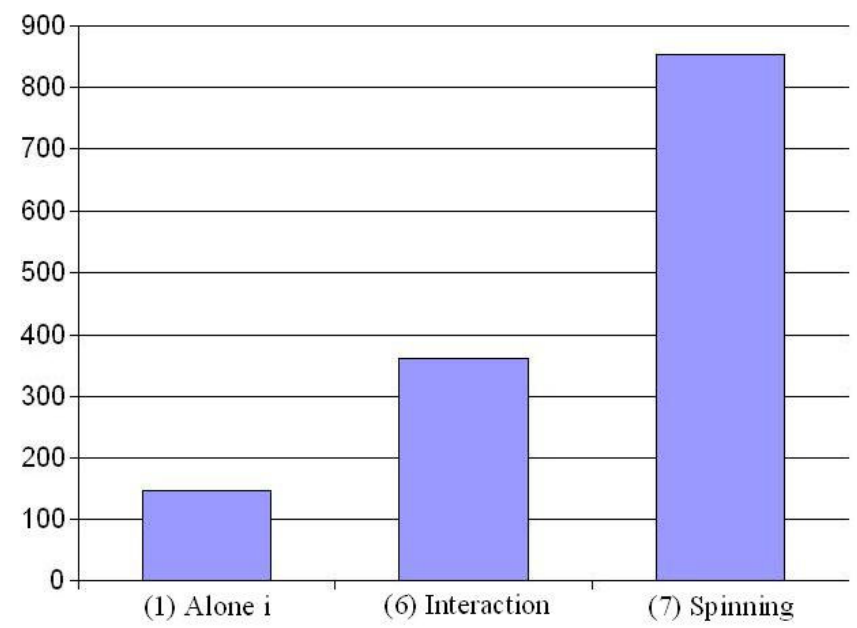

Fig. 4. Diagram showing the sum of opposite readings for the left/right tilt sensors when (1) Alone i, (6) Interaction and (7) Spinning over a 5 minutes trial. We can see that (7) Spinning produces the highest results.

However here, we are attempting to detect spinning whilst the robot is in motion.

Accelerometers, $\mathbf{X}, \mathbf{Y}$ and $\mathbf{Z}$ - Investigated were the averages, variance, sums, and the difference between axis readings compared to other the axis ${ }^{1}$ (i.e., the difference between $\mathrm{X}$ and $\mathrm{Y}$, the difference between $\mathrm{Y}$ and $\mathrm{Z}$, and the difference between $X$ and $Z$ ). A variety of graphs were also produced to visually investigate the readings (e.g., see Fig. 5 and Fig. 7).

\section{OBSERVATIONS AND RESUlTS}

Tilt Sensors Observations - Based on results shown in Fig. 4 , readings from tilt sensors can be used to detect that the robot is being spun. The most stable readings to detect spinning occur when Roball has no longitudinal motion (i.e., no forward or backward motion). Being still (not moving) whilst spinning enables the robot to be stable on the $\mathrm{Z}$ axis, which thus allows for right and left tilt sensors to indicate different readings e.g. (1) from the right tilt sensor and (2) from the left. This comes from the $\mathrm{G}$ force pushing them out. When the robot is spinning off a perfect $Z$ axis (i.e., tilted to one side), same tilt sensor readings (e.g., 2 from both sensors) are observed. Since Roball is programmed to always try to move either forward or backward, its internal plateau does not remain still while the robot is being spun, causing some misclassification. By summing the opposite readings for the left/right tilt sensors, a value of 895 is observed whilst the robot is moving (see Fig. 4), we would expect to see around 3000 when the robot is spinning but not moving (forward or backward motion). Therefore, even whilst moving, the tilt sensors can still give an indication whether the robot is spinning or not.

\footnotetext{
${ }^{1}$ When referring to the difference between two axis, e.g., $\mathrm{X}$ and $\mathrm{Z}$, we mean the calculation of $\mathrm{X}$ minus $\mathrm{Z}$.
}

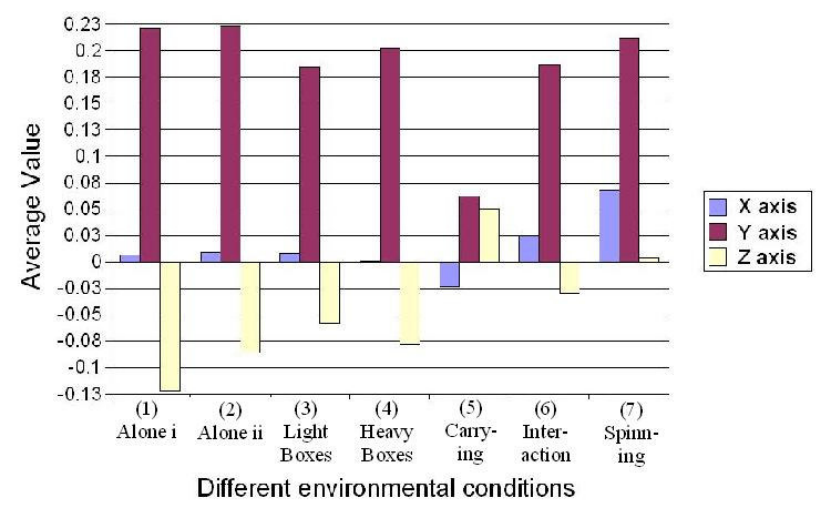

Fig. 5. Diagram showing the average reading of the $\mathrm{X}, \mathrm{Y}$ and $\mathrm{Z}$ axis for each of the seven different environmental conditions.

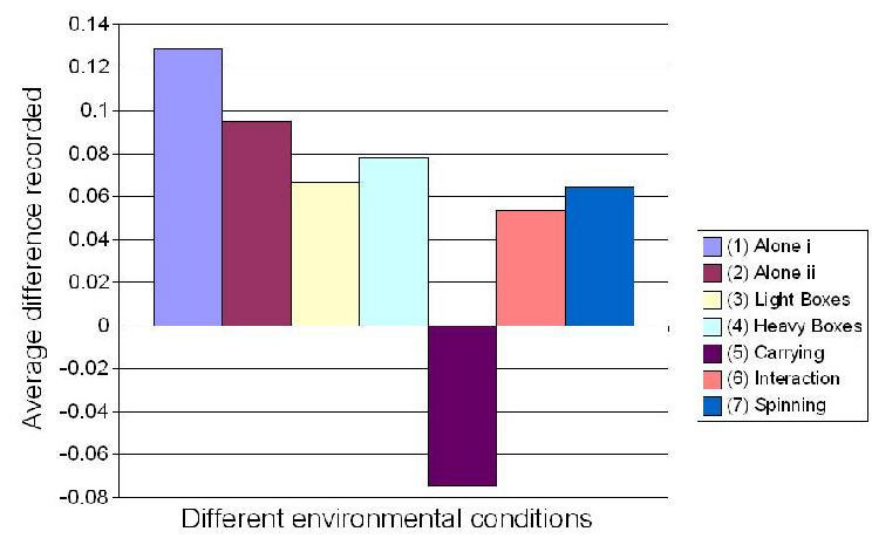

Fig. 6. Diagram showing the average reading of the difference between $X$ and $\mathrm{Z}$ axis for each of the seven different environmental conditions.

This information can be coupled along with other readings from accelerometers to give a clearer indication (see Fig. 8 and Fig. 10).

Accelerometers, $\mathbf{X}, \mathbf{Y}$ and $\mathrm{Z}$ Observations - Differences in the readings from the accelerometers can be clearly seen in line graphs (see Fig. 7, 8, 9, 10). Interaction and spinning can be recognized as very sharp jagged lines on the graphs (see Fig. 9 for an example of 'Interaction'). Without interaction (Alone), we observe large gaps between the lines of the different axis (see Fig. 7 for an example). Whilst being carried, we see consistently low $\mathrm{Y}$ axis readings (see Fig. 8). We have discovered two different ways of quantitatively analyzing the accelerometer readings that yield such results.

- Simply looking at the average reading for each of the three different axis, for each of the seven experimental conditions, can produce interesting information. We recorded the readings over the three separate experiment's conducted for each condition. We then worked out the average axis reading for each of these experiments and then finally took the average from the three experiments. 


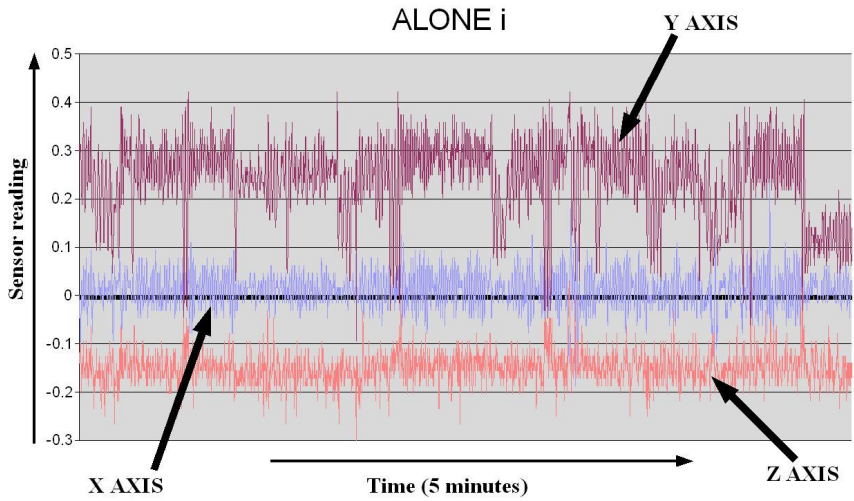

Fig. 7. Typical graph of sensor data from the accelerometers when Roball is in experimental condition (1) Alone i. Gaps between each axis can be seen and the axis rarely cross each other.

\section{CARRYING}

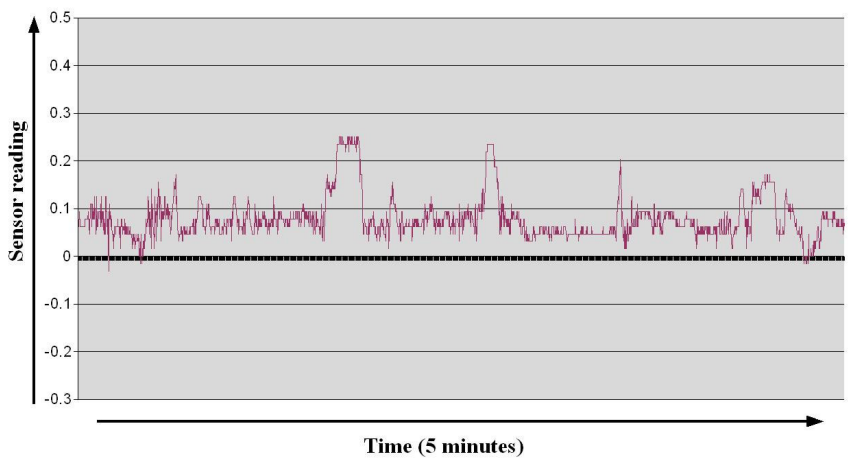

Fig. 8. Typical graph showing the unusually low $\mathrm{Y}$ axis sensor data (compared to the other conditions) from the accelerometers when Roball is in experimental condition (5) Carrying.

It appears that this analysis can indicate when the robot is being carried and also when it is spinning (spinning can further be confirmed by tilt sensor readings).

- Investigating the average difference (again over each of the experiments) between the varying accelerometer readings gives an indication as to whether the robot is 'Alone' and not receiving human interaction, or whether the robot is receiving interaction from a human. In particular, the average difference between $\mathrm{X}$ and $\mathrm{Z}$ has produced interesting results.

Based on such observations, analysis of accelerometers has yield the following results:

- (1) Alone i. Detecting when the robot is alone and in an unconfined space appears to be the easiest to recognize of all of the four ( 1 to 4 ) experimental conditions where the robot does not receive interaction from a human. Condition 1 produces the highest average difference between $\mathrm{X}$ and $\mathrm{Z}$ axis (0.13 shown in Fig. 6). Also this experimental condition shows the lowest negative reading on the $\mathrm{Z}$ axis (-0.12 shown in Fig. 5).

- (2) Alone ii. (3) Light boxes and (4) Heavy boxes. Despite being confined in a cluttered environment and

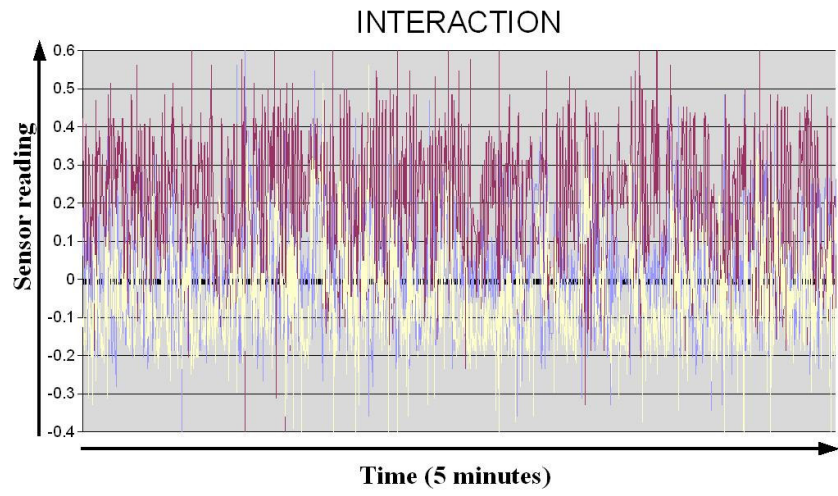

Fig. 9. Typical graph of the erratic $\mathrm{X}, \mathrm{Y}$ and $\mathrm{Z}$ axis sensor data from the accelerometers when Roball is in experimental condition (6) Interaction. All three axis readings show jagged readings that constantly cross with each other.

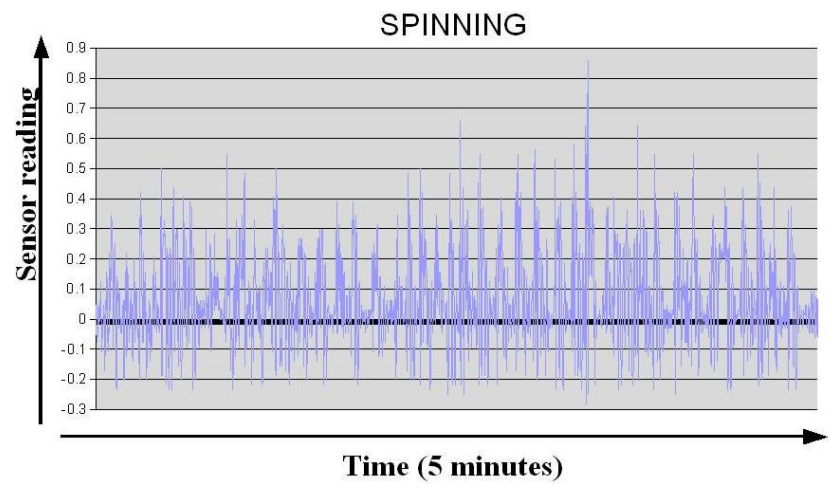

Fig. 10. Typical graph of $\mathrm{X}$ axis sensor data from the accelerometers when Roball is in experimental condition (7) Spinning. Spinning gives us the highest average reading for the $\mathrm{X}$ axis.

therefore banging against boxes or walls, Roball's $X$ and $\mathrm{Z}$ accelerometer readings still show a large difference during these conditions (see Fig. 6).

- (5) Carrying. We see very low average Y readings here (0.06 shown in Fig. 5). Also, this condition is the only time we see an average negative $\mathrm{X}$ axis reading $(-0.02$ shown in Fig. 5) and the only time we see a negative average for the difference between $\mathrm{X}$ and $\mathrm{Z}$ (see Fig. 6).

- (5) Carrying and (7) Spinning. Only during conditions 5 and 7 do we receive positive $\mathrm{Z}$ axis readings (see Fig. $5)$.

- (6) Interaction. When the robot is interacting with someone, we consistently see that the average difference between the readings of all of the accelerometers is lower than that of the other experimental conditions (without being negative). This can be visualized from the extremely jagged graph shown in Fig. 9, and also from the histogram graph of all of the experimental scenarios, shown by Fig. 6.

- (3) Light Boxes. This condition's readings are closer to the interaction condition (6) than any other. One possible explanation for this may be that at times when the robot 
hit light boxes, the boxes moved and therefore the robot did not stop and backup to avoid the box, but instead continued forward whilst still pushing and having contact with the box. This may have caused interference with the robot's sensor readings, similar to when the robot is receiving interaction.

\section{RECOGNIZING INTERACTION FROM A ROBOT'S PERSPECTIVE}

Based on the results from above, we have assigned some preliminary guidelines to create zones in which to classify the readings coming from the robot's sensors. These guidelines will give indications as to the robot's current environmental condition (e.g., receiving interaction, being carried). We hope to apply these zones in a planned future child-robot trial involving Roball. Our aim is to develop an adaptive algorithm onboard Roball that will recognize different environmental conditions. The algorithm will contain and be based on the following knowledge:

A Alone. If the average difference between the $\mathrm{X}$ and $\mathrm{Z}$ axis is above 0.07 , set current condition to 'Alone'.

$B$ Interaction. If the average difference between the $X$ and $\mathrm{Z}$ axis is below 0.05 but above zero, set current condition to 'Interaction'.

$\mathrm{C}$ Carrying. If the average difference between the $\mathrm{X}$ and $\mathrm{Z}$ axis is negative, set current condition to 'Carrying'.

$\mathrm{D}$ Spinning. If the average reading for the $\mathrm{Z}$ axis is positive and coupled with an average $\mathrm{Y}$ axis reading of above 0.08 , set condition to 'Spinning'; or if tilt sensors show different readings (see tilt sensor results) and the average reading for the $\mathrm{Z}$ axis is positive, set condition to 'Spinning'.

\section{DISCUSSION}

Our ultimate aim is to develop a system to allow adaptation of a robot to the interactions of children that are playing with it. Unlike the previous work [10], our intention is not to determine the personality type of the child, but to simply have the robot react and adapt to the type of interactions it is receiving. For example, the robot could adapt to general interactions, such as, being picked up or receiving rough and active interaction, thus making the robot's behavior more suited toward the individual interacting with it. The trials documented in this paper have been conducted to ascertain; what do the sensor readings produce in various situations the robot is encountering whilst in a human (child) populated environment? e.g., what do the accelerometers register when Roball is pushed? This gives us prior knowledge and understanding when attempting to analyze sensor readings from child-robot trials. If we are to achieve our ultimate aim of the robot adapting to the children, we must have a clear understanding and knowledge of what sensors register under varying conditions. The more data we collect from childrobot studies and further analysis we conduct, the greater our understanding will be. This will enable us to better categorize the different environmental conditions or interactions the robot

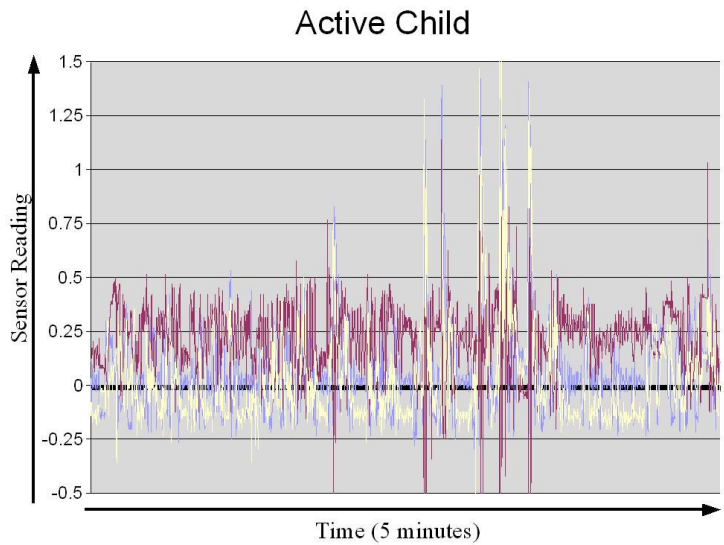

Fig. 11. Line graph of Roball's sensor readings when interacting with an active child. It is possible to see from erratic jagged lines that interaction occurred throughout most of the trial.

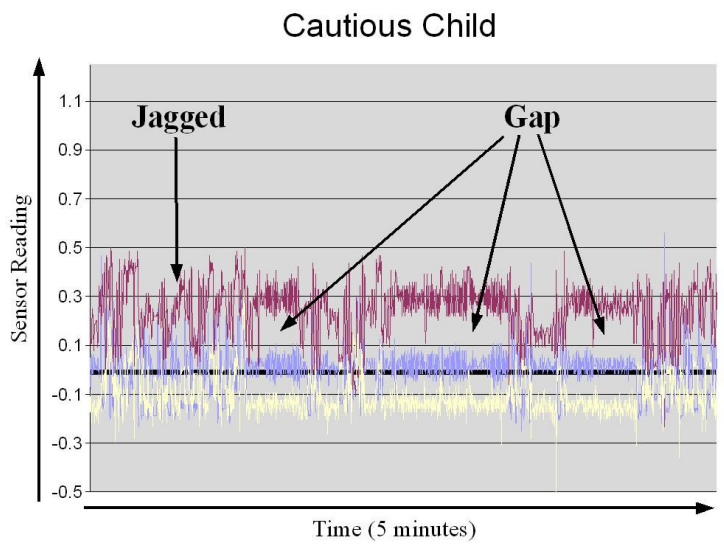

Fig. 12. Line graph of sensor readings when interacting with a cautious child. It is possible see that this child interacted with the robot most at the beginning of the trial, indicated by jagged lines. Further on gaps between the axis readings confirm lack of human interaction, or the robot being 'Alone'.

is experiencing. As we further our knowledge we hope in the future to be able to categorize more than the four categories listed in section $\mathrm{V}$.

As illustrated by Fig. 2, we have conducted trials at an elementary school and at a child's play group with children aged between five and seven years old. A pen was constructed with wooden planks. The children are asked to step inside the pen and to play with Roball. Trials were held over a four week period. Each trial lasted for four or five minutes. Sensors readings were recorded for the duration of the trial and video taped was used to verify the readings from the sensors. Initial results from the school trials are positive. Preliminary analysis appears to indicate that results described in this paper hold true when Roball is in a real life setting with children. We have observed the same type of readings as those recorded in the laboratory. When the robot is in a child populated environment but not receiving any interaction we see large gaps in the line graphs, as shown in Fig. 12. We also observe that interaction from the children is indeed indicated by two 


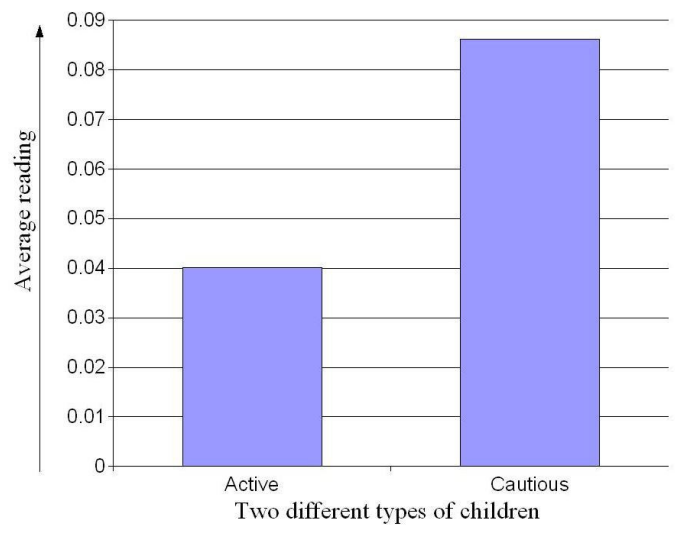

Fig. 13. Average difference between the $\mathrm{X}$ and $\mathrm{Z}$ axis for an active child and a cautious child.

factors: jagged, erratics lines on graphs, see Fig. 11 and low average $\mathrm{X}-\mathrm{Z}$ readings, see Fig. 13. Finally, we observe greater activity patterns from more active children as shown by Fig. 11 and 12.

This system has been tested under laboratory conditions, also, at a school and playgroup. We are now finishing analysis of the school and playgroup data and, developing an algorithm that will hopefully, not only automate the system of analyzing sensor data but, also allow the robot to adapt its behavior to various interactions.

\section{CONCLUSION}

From results obtained so far, it appears that it is possible to detect different environmental conditions through the analysis of proprioceptive sensors. This confirms previous work with a different platform that had navigational sensors [10], [11]. This suggests two things, that the use of proprioceptive and navigational sensors may surpass simply being used to traverse the environment, and also, that it is likely, that this system will transfer to other robots with other types of sensors. Using accelerometers and tilt sensors, we found the detection of carrying was the easiest, followed by spinning. Not so easy but still possible was detection of interaction from a person. Analysis does not require complex algorithms and it is hoped that guidelines created from the results in this paper will enable simple adaptation to the environmental conditions the robot is experiencing. Carrying out preliminary trials prior to conducting child-robot trials has certainly helped in understanding what sensor readings are created onboard Roball in various environmental conditions. This has given us a greater knowledge and awareness when we are analyzing the data from the school and play group trials. Future data analysis will be carried out over a much shorter time period rather than simply analyzing the whole trial. This is necessary as the different actions or conditions the robot is experiencing must be analyzed separately from each other so as not to cause confusion or mistake in analyzing the results. For example, we are currently working on a short time based algorithm that will detect carrying and spinning first, and if these conditions are found to be true no further analysis of the accelerometers will be carried out. If these conditions are found to be false, analysis of accelerometers will be carried out in an attempt to ascertain whether the robot is receiving any other form of interaction or whether it appears to be alone. We have presented initial guidelines so as to classify the readings coming from the onboard sensors into zones that will detect four main environmental conditions: (A) Alone, (B) Interaction, (C) Carrying and (D) Spinning. Our next step is to carry out further analysis of data from the completed trial at the school and playgroup, then to complete the algorithm that is currently being developed, this will automate the process of analyzing the sensor readings. Then we will conduct the planned child-robot trial at the Université de Sherbrooke with this algorithm onboard, hopefully allowing the robot to adapt its behavior to the child it is interacting with.

\section{ACKNOWLEDGMENT}

F. Michaud holds the Canada Research Chair (CRC) in Mobile Robotics and Autonomous Intelligent Systems. Support for this work is provided by the Canada Research Chair program and the Natural Sciences and Engineering Research Council of Canada.

\section{REFERENCES}

[1] T. Ito, "How children perceive robots", NEC Personal Robot Center http://www.incx.nec.co.jp/robot/english/univ/05/univ_e05.html, last accessed 06/10/04, 2003.

[2] B. Robins, K. Dautenhahn, R. te Boekhorst, A. Billard, "Effects of repeated exposure of a humanoid robot on children with autism", Universal Access and Assistive Technology (CWUAAT), pp. 225-236, 2004.

[3] S. Woods, K. Dautenhahn, J. Schultz, "Child and adults' perspectives on robot appearance" Proc. AISB'05 Symposium Robot Companions: Hard Problems and Open Challenges in Robot-Human Interaction, 2005.

[4] T. Kanda, T. Hirano, D. Eaton, H. Ishiguro "Interactive robots as social partners and peer tutors for children: A field trial", Human-Computer Interaction, pp. 61-84, 2004.

[5] T. Watanabe, R. Danbara, M. Okubo, "InterActor: Speech-Driven Embodied Interactive Actor", Proc. 11th International Workshop on Robot and Human Interactive Communication, Berlin, Germany, pp. 430-435, 2002.

[6] B. Jensen, G. Froidevaux, X. Greppin, A. Lorotte, L. Mayor, M. Meisser, G. Ramel, R. Siegwart, "The interactive autonomous mobile system RoboX", Proc. IEEE/RSJ International Conference on Intelligent Robots and Systems, pp. 1221-1227, 2002.

[7] D. Wada, T. Shibata, T. Saito, K. Tanie, "Robot assisted activity for elderly people and nurses at a day service center", Proc. IEEE International Conference on Robotics and Automation, Washington, DC, pp. 1416$1421,2002$.

[8] A. Billard, "Robota: Clever toy and educational tool", Robotics and Autonomous Systems, 2003, 42, pp. 259-269.

[9] Sony, http://www.aibo-europe.com, lasted accessed 06/10/04.

[10] T. Salter, K. Dautenhahn, R. te Boekhorst, "Learning about natural robothuman interaction styles", Robotics and Autonomous Systems, accepted for publication, 2005.

[11] T. Salter, K. Dautenhahn, R. te Boekhorst, "Robots moving out of the laboratory - Detecting interaction levels and human contact in noisy school environments", Proc. 13th IEEE International Workshop on Robot and Human Interactive Communication, Kurashiki, Okayama Japan, IEEE Press, pp. 563-568, 2004.

[12] T. Salter, K. Dautenhahn, "Guidelines for robot-human environments in therapy", Proc. 13th IEEE International Workshop on Robot and Human Interactive Communication, Kurashiki, Okayama Japan, IEEE Press, pp. 41-46, 2004

[13] F. Michaud, S. Caron, "Roball, the rolling robot", Autonomous Robots, 12(2), pp. 211-222, 2002. 